\title{
Formación de investigadores en medicina familiar: El modelo de tutorización en investigación Asesor-Tutor-Residente (ATR)
}

\author{
E. Raúl Ponce Rosas, Silvia Landgrave I báñez, Arnulfo E. I rigoyen Coria, Margarita Terán Trillo, \\ Francisco J. F. Gómez Clavelina, Miguel Ángel Fernández Ortega.
}

Departamento de Medicina Familiar, Facultad de Medicina, UNAM. México

El tu tor de la medicina de familia, los principios y caracterís ticas de la tutoría y la relación tu tor-residente son element os fundamentales para el des arrollo adecuado de los cursos de especialización de esta disciplina. La tutoría o tu torización en medicina familiar es la base de la docenciay se centra en la figura de una relación humana de enseñanza in dividualizada tutorresidente en la práctica asistencial, docente y de inves tigación. Los modelos de tutorización en medicina de familia proporcionan herramientas útiles para establecer una adecuada relación tu tor-residente. En este trabajo se des cribe el modelo de tu torización en inves tigación Asesor-Tutor-Residente, que se lleva a cabo en el Curs o de Especialización de Medicina Familiar en la Facultad de Medicina de la UNAM, México. El modelo de tu torización en inves tigación Aseso r-Tutor-Residente (ATR), es un paradigma de capacitación teórico-práctico en esta importante y fundamental área de la medicina de familia. El modelo ATR pretende motivar e interesar a tuto res y residentes para hacer inves tigación con base en la demostración y la supervisión fo mativa a través de la ases oría continua, reflexiva, planeada y sistematizada.

Palabras clave: Tutoría en investiga ción, medicin a fa miliar, mo delo a sesor-tutor-residente, docencia de la investiga ción.

Correspondencia:

E. Raúl Ponce Rosas

Coordinación de Investigación. Depto. de Medicina Familiar. Facultad de Medicina, UNAM. Circuito I nterior S/N (Detrás del Centro Médico Universitario)

Ciudad Universitaria, Distrito Federal, México cp. 04510

A partado postal 70-422 México, D.F. cp. 04510

Tel. (52 55)56 220060 • Fax: (52 55) 55502835

E-mail: ipr@servidor.unam.mx
Researchers training on family medicine: The tutoring model in investigation advisory-tutor-resident (ATR) “

The tutor of the family medicine, the principles and characteristics of the tutorship, and the relationship tutor-resident they are fundamental elements for the appropriate development of the cours es of specialization of this discipline. The tutorship or tutoring in family medicine are the base of the teaching and it is centered in the figure of a human relatio nship of teaching in dividu alized tu tor-resident in the assistance, educational practice and inves tigation. The tutoring models in family medicine provide useful tools to estab lis $h$ an app ropriate relationship tuto r-resident. In th is work is describe the tutoring model in inves tigation "advisory-tu tor-resident" (ATR) that is carried out in the Course of Specialization of Family Medicine in the Scho ol of Medicine of the UNAM, Mexico. The tuto ring model in investigation ATR, it is a theoretical-p ractical training paradigm in this important and fund amen tal area of the family medicine. The model ATR seeks to motivate and to interest tutors and residents to make investigation with bas e in the demonstration and the formative su pervision through the con tinuous, reflexive, planned and systematized consultant ship.

Key words: Tutorship in investigation, tutoring in research, family medicine, model advisor-tutor-resident (ATR), teaching of the investigation.

EI propósito de este trabajo es describir, analizar y aportar elementos que conduzcan a obtener los fundamentos teóricos, metodológicos y prácticos de la tutorización en las actividades de investigación de tutores y residentes en un programa educativo de posgrado (Especialización en Medicina Familiar). 
Las reuniones internacionales sobre Educación Médica realizadas en Edimburgo 1988, Lisboa 1988, Venecia 1989, Creta 1990, Bruselas 1992 y Edimburgo 19931,2, emitieron diversas recomendaciones que se relacionan con la tutorización en investigación en la medicina de familia; dichas recomendaciones se muestran en la tabla1. Como se observa, se considera orientar la labor de los docentes para que funcionen como asesores y tutores, y realicen asesoría o tutoría respectivamente

La tutoría o tutorización se ha reconocido como la base de la docencia de la medicina de familia y es una pieza clave en el programa docente de la especialidad ${ }^{3-13,18-20}$. De la misma forma, la tutorización orientada a la investiga ción es fundamental para lograr la consolidación de la medicina de familia a través de la investigación original en la disciplina.

También se puede observar que las recomendaciones mencionadas en las reuniones internacionales sobre Educación Médica, se encuentran directamente relacionadas con los principios vertidos en la pirámide de Miller para evaluar la competencia dínica ${ }^{1417}$.

Los niveles establecidos en la pirámide por orden de importancia de menor a mayor son: saber (knowledge), saber como (competence), mostrar como (performance) y hacer (action). Las características generales de cada nivel que se relacionan con la tutorización en investigación se muestran en la tabla 2.
Las recomendaciones en Educación Médica ${ }^{1,2}$, la pirámide de Miller ${ }^{1417}$, el reconocimiento de la figura del tutor en medicina familiar como pieza clave en los sistemas de instrucción actuales de la medicina ${ }^{3-13}$, la tutorización en medicina familiar ${ }^{8,13,18-20}$, la relación tutor-residente ${ }^{8-13}$ y el acuerdo de que la consolidación de la medicina familiar está relacionada con su propia contribución investigativa; constituyen la base del modelo para la enseñanza-aprendizaje de la investigación en medicina familiar que a continuación se expone.

El asesor es un profesor-investigador en medicina fami liar con conocimientos, habilidades, destrezas, valores, ética, competencias, reconocimiento, tradición y experien cia en actividades de investigación. En este modelo el asesor forma un grupo con el tutor y residente para planear, organizar, integrar y dirigir un proyecto de investigación interviniendo con mayor profundidad en el diseño, conducción, análisis y la presentación de un trabajo de investigación (tesis de fin de cursos de la especialización en medicina de familia). Durante este proceso se generan ciertas necesidades y actividades de investigación por niveles análogos a la pirámide de Miller ${ }^{14-17}$.

Nivel 1 (saber). Las áreas de conocimiento señaladas en la tabla 3 son la base para iniciar el proceso de tutorización en investigación en la medicina de familia; en este nivel es muy importante que el tutor posea un alto nivel

Tabla 1. Recomendaciones de las reuniones internacionales sobre educación médica, ${ }^{1,2}$ que se relacionan con la tutorización en investigación.

\begin{tabular}{|c|c|}
\hline Elementos del proceso educativo & Recomendaciones \\
\hline Curriculum & Debe basarse en competencias y debe incluir ética. \\
\hline Aprendizaje & $\begin{array}{l}\text { El énfasis debe cambiar de la manera pasiva al aprendizaje activo, fomento y perfección de habilidades, realización de lectu- } \\
\text { ras en pequeños grupos y aprendizaje independiente y promover la adquisición de hábitos de aprendizaje activo a lo largo } \\
\text { de la vida (conocimientos). }\end{array}$ \\
\hline Estrategias & $\begin{array}{l}\text { Se debe introducir el aprendizaje basado en problemas y debe incluir la medicina basada en evidencia; debe existir énfasis al } \\
\text { acercamiento científico (rigor metodológico). }\end{array}$ \\
\hline Evaluación & Debe basarse en competencias y valores sociales (actitudes). \\
\hline Docentes & $\begin{array}{l}\text { Deben ser entrenados como educadores (formación docente), debe evaluarse su desempeño (performance), debe recom- } \\
\text { pensarse la excelencia educativa (reconocimiento), deben actuar como asesores o tutores. }\end{array}$ \\
\hline Selección de alumnos & Debe ir mas allá de habilidades intelectuales (conocimientos), es decir, debe incluir y evaluar habilidades, actitudes y valores. \\
\hline
\end{tabular}

Tabla 2. Características generales de los niveles de la pirámide de Miller ${ }^{14-17}$ que se relacionan con la tutorización en investigación

\begin{tabular}{l|l}
\hline Niveles & Características \\
\hline $\begin{array}{l}\text { Nivel 1. } \\
\text { Saber (knowledge) }\end{array}$ & $\begin{array}{l}\text { De manera general, el saber lo conforma los conocimientos de la disciplina, los cuales se identifican con los objetivos de } \\
\text { aprendizaje de un prog rama educativo relacionados con el perfil profesional que se desea obtener y necesariamente vincula- } \\
\text { dos a las áreas de trabajo y competencias profesionales establecidas }\end{array}$ \\
\hline $\begin{array}{l}\text { Nivel } 2 . \\
\text { Saber como (competence) }\end{array}$ & $\begin{array}{l}\text { El saber como se fundamenta en la competencia, la cual es el g rado en que un sujeto puede utilizar sus conocimientos, } \\
\text { habilidades, aptitudes, actitudes y buen juicio (valores y ética) asociados a su profesión, para desempeñarla de manera eficaz } \\
\text { en todas las situaciones que corresponden al campo de su práctica }{ }^{1,1416} \text {. }\end{array}$ \\
\hline $\begin{array}{l}\text { Nivel 3. } \\
\text { Mostrar como (performance) }\end{array}$ & $\begin{array}{l}\text { El mostrar como implica el ser capaz de hacer algo a través del desempeño observado, la ejecución, realización, actuación o } \\
\text { funcionamiento y que ello pueda ser evaluado de alguna forma. }\end{array}$ \\
\hline Nivel 4. Hacer (action) & El hacer lo constituye la acción de una actividad o su práctica, evaluada a través de estándares establecidos previamente. \\
\hline
\end{tabular}


Tabla 3. Nivel 1 del Modelo de tutorización en investigación asesor-tutor-residente*

- Nivel 1 (saber). Está constituido fundamentalmente por las áreas de conocimientos que se consideran esenciales para iniciar el proceso de tutorización en investigación de la Medicina Familiar. Los aspectos específicos que desde nuestra experiencia son fundamentales y necesarios se describen a continuación:

1. Marco normativo de la Medicina Familiar como especialidad en el Reglamento General de Estudios de Posgrado (RGEP-UNAM) ${ }^{23}$ y el Plan Único de Especializaciones Médicas (PUEM-FAC.MED-UNAM) ${ }^{21}$

2. Historia, filosofía, fundamentos y desarrollo de la medicina familiar (contexto internacional y nacional).

3. Perfil del médico familiar (P UEM) ${ }^{21}$ y la Organización Mundial de Médicos de Familia (WONCA) ${ }^{2}$.

4. Áreas de trabajo del médico de familia (en el individuo, la familia y la comunidad en el contexto asistencial, docente y de investigación) ${ }^{24}$.

5. Áreas de competencias y atributos en la medicina familiar ${ }^{8}$.

6. Principios y características de la relación tutor-residente en la medicina familiar ${ }^{3-13,18-20}$.

7. Modelos de tutorización en medicina familiar ${ }^{8,13,18-20}$.

8. Modelos de formación docente (necesidades de formación docente y ritmo formativo) en el tutor de Medicina Familiar, ${ }^{4,819,20}$.

9. Metodología de la investigación, epidemiología, epidemiología clínica, bioestadística, administración básica, internet.

* Departamento de Medicina Familiar, Facultad de Medicina, UNAM, México.

Tabla 4. Nivel 2 del Modelo de tutorización en investigación asesor-tutor-residente*

- Nivel 2 (saber como). La base para ascender a este segundo nivel es el desear y querer hacer investigación. El tutor debe saber cómo incorporar la responsabilidad, la confianza mutua, el respeto personal y la comunicación clara y fluida en un ambiente de relaciones interpersonales sanas con crítica y autocrítica orientadas hacia el trabajo de investigación ${ }^{8}$.

\section{Habilidades}

1. Práctica de la lectura crítica, reflexiva y sistemática que incluya la simulación teórica.

2. Habilidad de perseverancia, practicando la constancia y dedicación al trabajo de investigación (tesis).

3. Habilidad de comprensión, conceptualización, razonamiento y juicio.

4. Desarrollo de la creatividad y la imaginación.

5. Practicar la comunicación clara, fluida y acertiva.

6. Capacidad del tutor para incentivar al residente.

Actitudes

1. Desear y querer hacer investigación.

2. Motivación e interés por la investigación.

3. Entusiasmo y estímulo en el binomio tutor-residente.

4. Confianza mutua entre tutor y residente.

5. Respeto personal, tolerancia y cooperación.

6. Flexibilidad y empatía mutuas.

7. Desarrollo de la crítica y autocrítica.

8. Percepción y convencimiento de la necesidad y utilidad de la investigación.

* Departamento de Medicina Familiar Facultad de Medicina, U NAM, México.

de competencia clínica y que paulatinamente adquiera los conocimientos que se han señalado, mediante un proceso de autoformación continuo a lo largo de su actividad como tutor y durante su trabajo en el curso de especialidad. No se trata que, de facto, el tutor maneje todas las áreas señaladas, sino que, de acuerdo a su propio ritmo formativo, interés y motivación personal, disponibilidad de tiempo, capacidad intelectual y orientación dirigida por el asesor en investigación, se capacite de forma integral para que inicie su trabajo de tutorización en investigación con el residente que se le asigne. Es necesario señalar que pueden existir tutores que ya poseen estos conocimientos, en ese caso ideal, serían los tutores idóneos para pasar al segundo nivel del modelo.

Es muy importante resaltar que la relación que exista entre el tutor y el residente es fundamental para la selección de los residentes que pueden potencialmente tener éxito en el modelo propuesto; esta selección se debe fundamentar en una evaluación de las siguientes características del residente realizada por el tutor: responsabilidad, disposición al trabajo en equipo, compromiso, interés y motivación por la investigación, conocimientos básicos de investigación y sus áreas que la auxilian. Estos elementos deben ser evaluados de manera objetiva y/o subjetiva por el tutor a través del conocimiento personal y el trabajo (actividades) que el residente realiza durante los primeros meses de su entrenamiento clínico. Por otra parte, es imprescindible que residentes y tutores manejen con cierta profundidad los conocimientos filosóficos y esenciales de la disciplina; estoes necesario para generar identidad a los trabajos de investigación que se produzcan, que pertenezcan a la medicina familiar y a su cuerpo de conocimientos con un abordaje propi o de la especialidad; esto constituirá la base para lograr una tradición investigadora.

Nivel 2 (saber como). En la tabla 4 se describen las características que deben desarrollarse con base en las habilidades y actitudes. El principal obstáculo en este nivel es percibir a la investigación como una obligación o imposición. Si bien el Programa Único de Especialidades Médicas de la Facultad de Medicina (PUEM) de la Universidad Nacional Autónoma de México UNAM ${ }^{21}$, señala que es un requisito el presentar un trabajo de investigación (tesis) al final del curso de especialización, 
Tabla 5. Nivel 3 del Modelo de tutorización en investigación asesor-tutor-residente*

- Nivel 3 (mostrar cómo). En este nivel se incorpora la figura del asesor en investigación formando parte del equipo (asesor-tutor-residente); se parte de un principio: el experto es el que mejor puede describir y mostrar cómo realiza su propio trabajo.

- El asesor utiliza todas las herramientas factibles y posibles para asesorar el proceso de investigación de acuerdo a su experiencia, capacidades y competencia.

- El asesor muestra cómo se trabaja en equipo de manera eficaz.

- El asesor muestra al tutor cómo se realiza el trabajo práctico de asesoría en investigación.

- El asesor capacita en investigación basándose en la acción, es decir, hace intervenir al tutor y al residente en forma participativa y activa.

- El asesor ejercita la simulación estadística y de investigación en situaciones reales, precisas y concretas.

- El asesor utiliza el aprendizaje basado en problemas y la medicina basada en evidencia.

- El asesor muestra el proceso activo de pasar de la lectura a la escritura.

- El asesor muestra la elaboración de mapas conceptuales para su uso en la investigación.

- El asesor muestra la elaboración de la matriz de consulta bibliográfica referencial y metodológ ica (como instrumento didáctico).

- El asesor muestra la construcción y uso del bibliorato en el proceso de investigación.

- El asesor coordina las actividades y participa de manera conjunta con el tutor y residente en la planeación y ejecución del proyecto de investigación.

- El asesor realiza una supervisión capacitante con el tutor y el residente con rigor científico, metodológico, estadístico y ético.

* Departamento de Medicina Familiar, Facultad de Medicina, UNAM, México.

Tabla 6. Nivel 4 del Modelo de tutorización en investigación asesor-tutor-residente*

- Nivel 4. (Hacer). Realización de productos de la acción o práctica de la investigación. Se favorece el aprendizaje a través de la supervisión activa y formativa, continua y capacitante realizada por el asesor a través de la demostración; fomentando la cultura de la evaluación y autoevaluación del tutor y el residente.

Participación en eventos académicos de la medicina familiar

- En este primer grupo se incluyen las conferencias, ponencias y la presentación de trabajos libres en cartel o en forma oral como productos directos de proyectos de investigación realizados por residentes y tutores.

Manuscritos autorizados (tesis)

- En este segundo g rupo se incluyen las tesis terminadas de los residentes y autorizadas por el profesor titular del curso de especialización.

Manuscritos aceptados para su publicación en revistas científicas con comité editorial

- Este tercer grupo incluye los ensayos, los artículos de revisión, los artículos de actualización y los trabajos de investigación originales. La preparación de este tipo de manuscritos exige compromiso, entrega, responsabilidad y ética entre el asesor, el tutor y el residente.

* Departamento de Medicina Familiar, Facultad de Medicina, UNAM, México.

el residente debe estar consciente y convencerse de que es necesario y útil para su formación como médico familiar (hacer la residencia para aprender).

En este segundo nivel la relación interpersonal que se establezca entre tutor y residente es fundamental para reconocer las habilidades y actitudes que posean y desarrollar las que no han logrado; la relación debe ser honesta y franca, con acertividad en el manejo de los conflictos interpersonales que se susciten entre ambos, buscando y orientando siempre el medio apropiado para el logro de sus objetivos de formación en investigación.

El trabajo del asesor en investigación en este nivel es mantener una cl ara y abierta comunicación con los tutores y residentes, los propósitos de esta comunicación se orientan hacia "detectar" fortalezas y debilidades en ellos que puedan influir en las actividades de investigaión de ambos; el asesor "hace" un diagnóstico de sus capacidades de investigación a través de entrevistas personales; orienta al tutor y al residente hacia aquellos conocimientos, habilidades, actitudes y val ores que deben poseer y ubica a ambos para que definan y reconozcan daramente ed panorama de la investigación dentro del campo y los principios de la Medicina Familiar (consejería en investigación).

Nivel 3 (mostrar como). El asesor se integra al equipo de trabajo como un guía-facilitador (tabla 5) y, junto con el tutor y el residente, planearán, orientarán y establecerán un plan de trabajo conjunto para realizar el trabajo de investigación durante los tres años de la residencia. Durante este período se deberá hacer un seguimiento y evaluación de las actividades y logros obtenidos no sólo cuantitativamente sino que, más importante, cualitativa mente (proceso de la enseñanza-aprendizaje de la docen cia de la investigación).

El asesor decide integrar o no a tutores y residentes a líneas sólidas y precisas de investigación en las cuales él es experto; otro esquema de trabajo, es que residente y tutor elijan su tema de investigación y el asesor, junto con ellos, le den el matiz e identidad propia hacia la medicina familiar.

En este nivel el asesor se percibe como un modelo de investigador activo, que conoce sus funciones como tal, que sabe asesorar y que tiene experiencia, que puede conducir y mostrar cómo se hace un proyecto de investigación, que conoce las líneas de investigación actuales de la disciplina, que tiene tradición investigadora y publicaciones (nacionales e internacionales), que posee competencias (conocimientos, habilidades, destrezas, actitudes, ética y valores en investigación), que es hábil y diestro en relaciones interpersonales y que sabe orientar y guiar en el proceso docente de la investigación ${ }^{22}$. Estas características suponen al asesor ideal, en la práctica, puede ser fac- 
tible que esto se presente o no; sin embargo, se necesita además una abierta disposición y deseo para enseñar la investigación por parte del asesor, con actitud positiva, tolerante y perseverante, además de apoyo logístico y de gestión administrativa de las autoridades educativas.

Nivel 4 (hacer). Se proponen tres tipos de productos (tabla 6):

\section{A. Participación en eventos académicos de la medicina familiar}

En estas actividades el asesor guía y apoya con su experiencia a tutores y residentes para planear y estructurar, de acuerdo a los requisitos de presentación de los eventos, los manuscritos y su contenido, materiales, uso adecuado del tiempo y estilo de presentación.

En este nivel es importante analizar y evaluar el trabajo activo de tutores y residentes, considerando además las capaci dades y limitaciones de ellos, es decir, en conjunto, se intercambian ideas claras y predisas decómo ha sido el trabajo para lograr un producto final que será presentado y no ser solamente un espectador de este proceso.

La reflexión, la crítica y la autocrítica son muy importantes en el grupo, aprendiendo de los errores y aciertos, de la propia experiencia de participar en este tipo de eventos y valorar la confianza que se adquiere. Estas acciones promueven la valoración de la integración y adaptación del equipo de trabajo, por lo que, se debe estimular y motivar a tutores y residentes para que participen siempre durante los tres años de la residencia.

La participación de los residentes debe ir aumentando paulatinamente en su complejidad, es decir, inicialmente (en el primer año), pueden presentar el marco de referencia de su proyecto, experiencias de trabajo, propuestas, protocolos de investigación, etc; posteriormente (segundo año), proyectos de investigación en proceso o con resultados preliminares, revisiones bibliográficas actualizadas sobre sus trabajos de investigación, revisiones y experiencias sobre temáticas importantes de la clínica y la docencia en Medicina Familiar. Finalmente (tercer año), pueden presentar los resultados definitivos de sus proyectos y las conclusiones a las que llegaron, trabajos originales, propuestas metodológicas dínicas y docentes fundamentadas con rigor científico y ético, temáticas en los que el tutor y/o residente se consideran expertos con cierta profundidad "desdela medicina de familia".

Por último, la partiajpación del tutor deberá aumentar paulatinamente, cada vez con mayor involucramiento en las diferentes actividades que real iza el residentey coordina y supervisa el asesor, se trata de adqui rir capacidades y habilidades que enriquezcan las competencias del tutor; el asesor pondrá énfasis en el rigor, los val ores y la ética.

\section{B. Manuscritos autorizados (tesis)}

Las autoridades educativas deberán estar enteradas oficialmente (desde que se inician las actividades conjun- tas), de que asesor, tutor y residente realizaron un trabajo de investigación para presentarlo como trabajo de fin de cursos (tesis). Dichas autoridades deben confiar y validar que la asesoría proporcionada por el asesor es de gran calidad técnica-metodológica, con rigor científico y ético, que ha sido planeada y ejecutada de acuerdo a las necesidades propias del tutor pero principalmente del residente El producto final (la tesis) representa no sólo un requisito, sino también un instrumento de enseñanza-aprendiza je dela investigación en la disciplina.

Las autoridades educativas también deben comprender y aceptar que durante el proceso de tutorización en investigación entre los tres el ementos humanos del modeIo, (asesor-tutor-residente), se presenta una relación muy particular, en la cual existen actitudes, habilidades, valores, ética y competencias que se van desarrollando paulatinamente, las cuales se van adquiriendo en grados diferentes por el tutor y el residente; es decir, no hay garantía de realizar tesis excepcionales en su calidad, sino que depende de las propias circunstancias que se presentan durante los tres años del curso de especial ización. No es lo mismo iniciar un trabajo de tesis a partir del tercer año de la residencia, que hacerlo en el segundo o el primero; tampoco es lo mismo iniciar una tutoría de investigación con un tutor inexperto, con poco tiempo como docente, que nunca ha realizado una investigación formal y rigurosa, que hacerlo con un tutor con experiencia docente y de investigación. Las condiciones de ventajas o desventajas son obvias y es aquí cuando emerge importantemente la entrevista que el asesor realiza antes de iniciar el proceso de tutorización.

Finalmente, las autoridades deben confiar y validar que el producto final (la tesis), no requiere "pasar acredi taciones o revisiones" realizadas por personas que nunca supieron que se trabajó en equipo de manera planeada, organizada y sistematizada para lograr el objetivo final, es decir, el valor agregado que proporciona la tutoría en investi gación, es sinónimo de calidad total y excelencia en el ámbito educativo de la investigación.

Con estos principios expuestos, la autorización de tesis prácticamente debe darse de manera automática cuando el asesor y el tutor han firmado el trabajo final; el profesor titular y demás autoridades simplemente deberán autorizarla administrativamente.

\section{Manuscritos aceptados para su publicación en revistas científicas con comité editorial}

La experiencia del asesor (sobre todo si ya tiene publicaciones nacionales e internacionales como primer autor), proporciona una ayuda de gran valor ya que conoce con precisión los detalles científicos, éticos, técnicos y administrativos que se deben seguir en el proceso de escribir un comunicado científico. De igual forma, la experiencia que también tenga el tutor en lo que se refiere a tener publicaciones como primer autor o coautor coadyuva a la realización de un manuscrito. 
El asesor no debe hacerse responsable total del comunicado, puede guiar, sugerir y aportar ideas, la organización, el estilo, las características de forma y de fondo que Ilevará el escrito; utilizando todas las herramientas posibles que él domina para guiar el formato final de acuerdo a los requisitos y normas de la revista el egida.

El objetivo que el asesor persigue es que el tutor y residente se esfuercen en escribir, para librar ese gran obstáculo que representa para muchos "el pasar de la lec tura a la escritura"; la única forma de aprender a escribir un artículo para revista, es escribiéndolo; si el residente y/o el tutor no lo hacen no podrán avanzar ni crecer en sus actividades de investigación.

La tarea del asesor es la de facilitar el aprendizaje del tutor y residente sobre la manera de escribir los comunicados de investigación, pero es necesario que sea de forma participativa, tripartita, para que tutor y residente adquieran y posean en un proceso de autoformación posterior, la competitividad e individualidad para hacer investigación original por sí mismos; esto conducirá a que el apoyo y ayuda del asesor será cada vez menor en frecuencia e intensidad y más bien, estarán en la posibilidad de integrarse a grupos y redes de investigadores de la Medicina de Familia.

\section{REFLEXIONES F INAL ES}

El modelo de tutorización en investigación asesortutor-residente descrito en este trabajo, se aplica en el Curso de Especialización de Medicina Familiar, en el Departamento de Medicina Familiar de la Facultad de Medicina de la Universidad Nacional Autónoma de México, desde agosto de 2000. Los resultados parciales con dos generaciones de residentes y un grupo de tutores son hasta ahora muy satisfactorios. Una de las principales características del modelo ATR parece ser su capacidad para motivar e interesar a tutores y residentes para hacer investigación; con base en la demostración (hacer), la supervisión capacitante y formativa, la asesoría continua, reflexiva, facilitadora, planeada y sistematizada que implica el necesario seguimiento, evaluación cualitativa de lo realizado y lo que falta por hacer y aprender en cada proyecto, individualizando el proceso con cada tutor y su residente

También se han establecido algunos valores entre el asesor, tutor y residente considerados fundamentales para facilitar el trabajo de investigación: responsabilidad, compromiso, constancia, honestidad, tolerancia, perseverancia, flexibilidad, respeto y cooperación. Estos valores y las características de los modelos de tutorización, así como los princi pios de la relación interpersonal tutor-residente son la base de este paradigma de capacitación teórico-práctica en la investigación de la medicina de familia en nuestro México.

\section{AGRADECIMIENTO}

A Raúl Ponce Pérez por su presencia y ayuda para realizar este trabajo. Esta investigación se ha realizado con el apoyo financiero del Departamento de Medicina Familiar de la Facultad de Medicina de la UNAM, México.

\section{REFERENCIAS BIBLIO GRÁFICAS}

1. García BM. Medical education in the light of the World $\mathrm{H}$ ealth Organization. Health for all strategy and the European U nion. Med E du 1995; 29(1): 3-12.

2. Rosinski EF. A generic definition of compency-based education. American J ournal of Pharmaceutical Education 1975. Citado en: García BM. Medical education in the light of the World Health Organization. Health for all strategy and the European Union. Med Edu 1995; 29(1):3-12.

3. Bonal PP, Gil GV, Martín ZA, Pinto HA. La medicina de familia como área de conocimiento. A ten P rimaria 1999; 23(3): 151-174.

4. Saura LIJ, Quirós BC, Molina DF. ¿Cómo llevar a cabo la tutorización de residentes en medicina familiar y comunitaria?. Aten Primaria 1994; 13(3): 103-104.

5. Saura LIJ . La evaluación de los residentes de medicina de familia y comunitaria. Aten Primaria 1995; 16(4): 179-180.

6. Saura LIJ. “EI manual del tutor" de atención primaria. Aten Primaria 1996; 17(3): 177-178.

7. Leal HM, Saura LIJ , López PM, De la Viesca CS, Ferrer MA, García MMM. Investigación en la acción. Cómo facilitar la incorporación de los residentes de MFyC al centro de salud. Aten Primaria 1996; 18 (10): 571-576.

8. Saura LIJ , Leal HM. Docencia posgrado: factores condicionantes de la relación tutor-residente en medicina familiar. Aten Primaria 1997; 20(9): 511-518.

9. Gayoso DP, Viana ZC, García GJ, Tojal del Casero F. Los tutores de medicina de familia: actitudes y actividades de tutorización. Aten Primaria 1995; 15(8): 481-486.

10. Oller CM, Vila CM, Asens MN, Castellanos DRM, Parra HL, Sierra AY. Autoaprendizaje o tutorización. Análisis cualitativa de las sesiones dínicas de residentes y tutores en un centro de salud. $X$ J ornadas Docentes de Medicina de Familia y Comunitaria. Aten Primaria 2000; 26 (sup. 1): 81.

11. Saura LIJ . ¿Cómo quieren los residentes que sean sus tutores?. IX J ornadas Docentes de Medicina de Familia y Comunitaria. Aten Primaria 1999; 24 (sup. 2): 43-44.

12. L eiva FF, Prados TD. Evaluación y mejora de la calidad en la aplicación de programa docente en MF yC: EI taller de tutores como instrumento de detección de problemas y diseño de medidas correctoras. VI II J ornadas Docentes de Medicina de Familia y Comunitaria. Aten Primaria 1998; 22 (sup. 1): 42-43.

13. Molina DF (Coordinador). Relación humana residente-tutor. VIII J ornadas Docentes de Medicina de Familia y Comunitaria. Aten Primaria 1998; 22 (sup. 1): 39.

14. Miller G. The assessment of dinical skills/competence/performance. Acad Med 1990; 65:S63-S67.

15. Blay PC. Evaluación de la competencia profesional: ¿están cambiando los tiempos?. Aten Primaria 1995; 16(1): 2-4.

16. Esteve RM. Competencias y desarrollo profesional. Educación Médica 1999; 2(2): 66-69.

17. Cots YJ M.Sistemas de acreditación de competencia clínica de los médicos de familia. Aten Primaria 2000; 26(suppl 1): 158-159.

18. Sant AE, Casajuana BJ , Botinas MN, Altirriva VJ, Vila CMA. Tutorización activa continua: una metodología para intentar ser tutor durante los tres años VIII J ornadas Docentes de Medicina Familiar y Comunitaria. Aten Primaria 1998; 22(suppl 1): 43.

19. Redondo SJ M, Aliaga MA. EI tutor novato: una propuesta racional. IX Jornadas Docentes de Medicina Familiar y Comunitaria. Aten Primaria 1999; 24( suppl 2): 41-42.

20. Turabián J L, Pérez FB. Avances en metodología docente. IX Jornadas Docentes de Medicina Familiar y Comunitaria. Aten Primaria 1999, 24(suppl 2): 42-43.

21. Plan Ú nico de Especializaciones Médicas (PUEM). Medicina Familiar. Facultad de Medicina, UNAM. Segunda edición, México 1998.

22. Valarino $E$, Meneses $R$, Yáber $G$, Pujol L. Optimización de la gerencia de investigación en postgrado. Rev Latinoamericana de Psicología 1996; 28(1): 63-82.

23. Reglamento General de Estudios de Posgrado. Universidad Nacional Autónoma de México. México 1996.

24. Gallo VFJ, Altisent TR, Díez EJ, Fernández SC, Foz i Gill G, Granados MMI cols. Perfil profesional del médic de familia. Aten Primaria 1999;23(4): 236-248. 